\title{
The kynurenine:tryptophan ratio as a predictor of incident type 2 diabetes mellitus in individuals with coronary artery disease
}

\author{
Eirik W. Rebnord ${ }^{1,2,3} \cdot$ Elin Strand $^{1}$ - Øivind Midttun ${ }^{4}$ - Gard F.T. Svingen ${ }^{3}$ • \\ Monika H.E. Christensen ${ }^{1,5}$ • Per M. Ueland ${ }^{1,6}$. Gunnar Mellgren ${ }^{1,2,7}$. \\ Pål R. Njølstad ${ }^{1,2,8}$ • Grethe S. Tell ${ }^{9}$ - Ottar K. Nygård ${ }^{1,2,3}$ • Eva R. Pedersen ${ }^{2,3}$
}

Received: 9 January 2017 / Accepted: 15 May 2017 / Published online: 13 June 2017

(C) The Author(s) 2017. This article is an open access publication

\begin{abstract}
Aims/hypothesis The tryptophan metabolite kynurenine has potent immune modulatory and vasoactive properties. Experimental data implicate kynurenine in obesity-related morbidities. Epidemiological studies are, however, sparse. We evaluated associations of the plasma and urine kynurenine:tryptophan ratio (KTR) to incident type 2 diabetes.

Methods We followed 2519 individuals with coronary artery disease (CAD; 73.1\% men) without diabetes at baseline for a median of 7.6 years, during which $173(6.9 \%)$ new incidences of type 2 diabetes were identified. Multivariate Cox regression analyses were applied to investigate the prospective relationships of plasma and urine KTR with new onset type 2 diabetes.

Results At inclusion, mean (SD) age was 61.3 (10.4) years, BMI was $25.9(3.71) \mathrm{kg} / \mathrm{m}^{2}$ and median (interquartile range) $\mathrm{HbA}_{1 \mathrm{c}}$ was $5.6 \%(5.0 \%-6.0 \%)(38[31-42] \mathrm{mmol} / \mathrm{mol})$. Plasma KTR was not significantly related to type 2 diabetes risk. By contrast, urine KTR showed a strong positive associ-

Electronic supplementary material The online version of this article (doi:10.1007/s00125-017-4329-9) contains peer-reviewed but unedited supplementary material, which is available to authorised users.
\end{abstract}

ation. Comparing quartile 4 with quartile 1, the HRs (95\% CIs) were $2.59(1.56,4.30)$ and $2.35(1.39,3.96)$ in the ageand sex-adjusted and multivariate models, respectively. Conclusions/interpretation Urine KTR is a strong predictor of incident type 2 diabetes in individuals with CAD. Potential clinical implications and possible pathogenic roles of renal kynurenine excretion in type 2 diabetes development should be further elucidated.

Keywords Amino acid metabolism · Biomarkers · Clinical epidemiology · Type 2 diabetes

$\begin{array}{ll}\text { Abbreviations } \\ \text { AIC } & \text { Akaike's information criterion } \\ \text { Apo } & \text { Apolipoprotein } \\ \text { CAD } & \text { Coronary artery disease } \\ \text { CRP } & \text { C-reactive protein } \\ \text { CVD } & \text { Cardiovascular disease } \\ \text { FE } & \text { Fractional kidney excretion }\end{array}$

Eirik W. Rebnord

Eirik.Rebnord@gmail.com

1 Department of Clinical Science, University of Bergen, Bergen, Norway

2 KG Jebsen Centre for Diabetes Research, University of Bergen, Bergen, Norway

3 Department of Heart Disease, Haukeland University Hospital, Jonas Lies vei 65, 5021 Bergen, Norway

4 Bevital, Bergen, Norway
5 Department of Medicine, Haukeland University Hospital, Bergen, Norway

6 Laboratory of Clinical Biochemistry, Haukeland University Hospital, Bergen, Norway

7 Hormone Laboratory, Haukeland University Hospital, Bergen, Norway

8 Department of Pediatrics, Haukeland University Hospital, Bergen, Norway Bergen, Bergen, Norway
9 Department of Global Public Health and Primary Care, University of 


$\begin{array}{ll}\text { IDO1 } & \text { Indoleamine 2,3-dioxygenase } \\ \text { IQR } & \text { Interquartile range } \\ \text { KTR } & \text { Kynurenine:tryptophan ratio } \\ \text { MetS } & \text { Metabolic syndrome } \\ \text { NO } & \text { Nitric oxide } \\ \text { NRI } & \text { Net reclassification improvement } \\ \text { Q } & \text { Quartile } \\ \text { TDO } & \text { Tryptophan 2,3-dioxygenase } \\ \text { WENBIT } & \text { Western Norway B Vitamin Intervention Trial }\end{array}$

\section{Introduction}

Type 2 diabetes mellitus is a chronic and slowly evolving disease characterised by impaired insulin-mediated glucose uptake in peripheral tissues and a failure of the insulin secreting capacity of the pancreas [1]. While traditionally considered primarily a disorder of glucose homeostasis, it is now recognised that type 2 diabetes is associated with profound metabolic dysfunction including amino acid metabolism [1]. Further, type 2 diabetes is characterised by systemic lowgrade inflammation, affecting the adipose tissue, liver and pancreas, as well as the kidneys [2].

Degradation of the essential amino acid tryptophan is closely related to immune activation. Induced by the proinflammatory cytokine IFN- $\gamma$, the enzyme indoleamine 2,3dioxygenase (IDO1) catalyses the first and rate-limiting step of tryptophan catabolism through the kynurenine pathway [3]. Circulating levels of the metabolite kynurenine are also influenced by dietary tryptophan intake [4]. Hence, the kynurenine:tryptophan ratio (KTR) provides a more appropriate measure of tryptophan catabolism than the absolute concentration of kynurenine. In addition to reflecting the activity of the IDO1 enzyme, plasma KTR is, along with the pteridine derivative neopterin [5], a reliable indicator of IFN- $\gamma$ mediated immune activation $[3,4]$. Interestingly, IFN- $\gamma$ has been implicated in the pathogenesis of insulin resistance [5-7] and IDO1 induction has well-characterised immunomodulatory effects [8]. Moreover, kynurenine has been identified as an endothelium-derived vasodilator $[9,10]$. In experimental models, this metabolite $[11,12]$ and its downstream intermediates kynurenic acid [11, 13, 14] and xanthurenic acid [14] have been implicated in the pathogenesis of type 2 diabetes. Further, several plasma metabolites from the kynurenine pathway correlate with insulin resistance [15-17] and other phenotypes of the metabolic syndrome (MetS) [18]. Urinary excretion of kynurenine was increased in individuals with type 2 diabetes [19]. However, the prospective association of kynurenine pathway activation to diabetes development has not been evaluated. Therefore, we explored plasma and urine KTR as predictors of new onset type 2 diabetes in a cohort of individuals with suspected or verified stable coronary artery disease (CAD).

\section{Methods}

Study population The source population has been described in detail elsewhere [20]. Briefly, it included 4164 adults, of whom 3096 were subsequently included in the Western Norway B Vitamin Intervention Trial (WENBIT) (https:// ClinicalTrials.gov/ identifier: NCT00354081). The participants underwent elective coronary angiography at two Norwegian university hospitals between 2000 and 2004. For the prospective analyses, we excluded 496 individuals with a self-reported diagnosis of diabetes mellitus at baseline. We additionally omitted 42 individuals with missing $\mathrm{HbA}_{1 \mathrm{c}}$ records and 1107 individuals with $\mathrm{HbA}_{1 \mathrm{c}} \geq 6.5 \%(\geq 48 \mathrm{mmol} / \mathrm{mol})$, fasting glucose $\geq 7.0 \mathrm{mmol} / 1$ or non-fasting glucose $\geq 11.1$ $\mathrm{mmol} / \mathrm{l}$. Of the 2519 eligible individuals included in the follow-up analyses, 2263 provided baseline urine samples (electronic supplementary material [ESM] Fig. 1). The study fulfilled the principles of the Declaration of Helsinki and was approved by the regional Committee for Medical and Health Research Ethics (approval number 2010/1880) and the Norwegian Data Protection Authority. All participants provided written informed consent.

Baseline data The procedures for collection of demographic, clinical and biochemical baseline characteristics have been described in detail elsewhere [20]. Participants were classified with $\mathrm{CAD}$ if coronary angiography revealed at least one significant stenosis (defined as $\geq 50 \%$ luminal narrowing in the main coronary arteries or major side branches). Spot urine samples were collected by the participants at home on the day of admission to hospital. Venous samples were obtained at a clinical examination before or immediately after coronary angiography. Participants reporting no intake of food or beverages during the last $6 \mathrm{~h}$ prior to sampling were defined as fasting. All plasma, serum and spot urine samples for the biobank were frozen at $-80^{\circ} \mathrm{C}$ until later analysed by the Bevital laboratory (www.bevital.no). Plasma levels of kynurenine and tryptophan were measured by liquid chromatography-tandem MS, whereas the corresponding urine concentrations were analysed by GC-MS/MS. The lower limits of detection and coefficients of variability have been reported elsewhere [19, 21]. eGFR, $\mathrm{HbA}_{1 \mathrm{c}}$, serum lipoproteins and C-reactive protein (CRP) were calculated or measured as previously described [22]. Concentrations of individual compounds in urine were given per mol creatinine to correct for dilution. Fractional kidney excretion (FE), defined as the fraction of analyte filtered in the glomerulus that is excreted in urine, was calculated using the formula:

$\mathrm{FE}_{\mathrm{S}}=\left([\mathrm{S}]_{\text {urine }} \times\left[\right.\right.$ creatinine $\left._{\text {plasma }}\right) /\left([\mathrm{S}]_{\text {plasma }} \times[\text { creatinine }]_{\text {urine }}\right)$,

where S denotes kynurenine or tryptophan. HOMA2-IR and, beta cell function were calculated based on plasma glucose 
and serum $C$-peptide in a subgroup of fasting participants $(n=607)[23]$.

Study endpoints Information on incident type 2 diabetes was collected until 31 December 2009. We identified new diagnoses of type 2 diabetes by linkage to the Norwegian Prescription Database (NorPD, www.norpd.no). This is a national registry containing data on all dispensed drugs at outpatient pharmacies in Norway. We classified participants as having incident type 2 diabetes when receiving a first prescription of an oral glucose-lowering drug or insulin (anatomical therapeutic chemical classification system code A10). Participants were also identified with type 2 diabetes when diagnosed according to the ICD-10 (codes E11-E14; www. who.int/classifications/icd/en/) on their discharge summary following admission to a Norwegian hospital. The hospital data were obtained from the Cardiovascular Disease in Norway (CVDNOR) project (www.cvdnor.no) [24]. For the subset included in WENBIT ( $n=1712,68.0 \%)$, information on new onset type 2 diabetes was also obtained from selfreports and verified by glucose measurements during in-trial follow-up (2000-2005) [20].

Statistical analyses Variables were reported as counts (\%), means (SD) or medians (interquartile range [IQR]), as appropriate. Differences in baseline characteristics according to diabetes status were evaluated using ANOVA and $\chi^{2}$ analyses for continuous and categorical variables, respectively. All non-normally distributed variables were natural $\log \left(\log _{e}\right)$ transformed before being used in parametric tests.

We applied multivariate linear regression to identify covariates associated with plasma and urine KTR. In order to avoid multicollinearity not all variables from Table 1 were included in the model. The results are reported as $R^{2}$ for models and standardised $\beta$ coefficients for individual variables.

Statistical power was evaluated on the basis of a two-sided $t$ test. At $\alpha=0.05$, we had a power of $100 \%$ to detect a difference of at least $15 \%(\geq 3.8 \mathrm{nmol} / \mu \mathrm{mol})$ of baseline mean plasma KTR levels between participants with and without incident type 2 diabetes. For baseline urine KTR, power was $81 \%$ to detect a $15 \%$ difference $(\geq 6.5 \mathrm{nmol} / \mu \mathrm{mol})$ of mean levels.

We calculated HRs and 95\% CIs for incident type 2 diabetes by Cox regression, reported per SD ( $\log _{\mathrm{e}}$ transformed) increase and for quartile (Q) 4 vs Q1 of urine and plasma KTR. The simple model was adjusted for age and sex. Additional covariates for the multivariate model were selected based on clinical relevance and included: BMI, eGFR, CRP, $\mathrm{HbA}_{1 \mathrm{c}}$, serum triacylglycerol, apolipoprotein (Apo) A-1, urine albumin:creatinine ratio, and the use of loop diuretics, ACE inhibitors or angiotensin II receptor blockers, statins and $\beta$-blockers. Visual inspection of survival plots did not suggest deviation from proportionality. Further, the tests for proportional hazards with Schoenfeld residuals gave $p$ values of $\geq 0.27$. Hence, we found no evidence of violation of the model assumptions.

There were $408(16.2 \%)$ and $256(10.2 \%)$ missing records for urine albumin:creatinine ratio and urine KTR, respectively. For all other covariates of the multivariate model the number of missing records was $\leq 3(\leq 0.1 \%)$. Missing data were handled by listwise deletion. In secondary Cox regression, we performed multiple multivariate imputation under the assumption of missing at random. Using the fully conditional specification (iterative Markov chain Monte Carlo) method [25], 20 imputed datasets were created. All covariates of the multivariate Cox model including plasma and urine KTR, the cumulative hazard rate (Nelson-Aalen estimator) and outcome variable (dichotomous) were included in the imputation model.

Subgroup analyses were performed for predefined categories of categorical variables, or according to median values of continuous variables. The following covariates were included: age, sex, BMI, presence of significant $\mathrm{CAD}$ at coronary angiography, eGFR, CRP, $\mathrm{HbA}_{1 \mathrm{c}}$, serum triacylglycerol, ApoA-1 and urine albumin:creatinine. We tested effect modifications by adding interaction product terms to the models. The Benjamini-Hochberg adjustment was applied to correct for false discovery rate. However, because the subgroup analyses were planned and performed in order to facilitate the interpretation of the overall study results, $p$ values for interaction are not reported adjusted for multiple comparisons in Fig. 2.

The relationships for plasma and urine KTR with incident type 2 diabetes were visualised by a $4 d f$ smoothing spline fit in multivariate Cox regression models [26]. We compared model fit using Akaike's information criterion (AIC) and explored model discrimination by calculating $\mathrm{C}$ statistics. By determining continuous net reclassification improvement (NRI >0) [27], we evaluated whether urine KTR improved risk classification of participants when added to the multivariate model.

All tests are two-tailed with a significance level set to 0.05 . The statistical analyses were performed using SPSS Statistics version 23 (IBM, Armonk, NY, USA), SamplePower 2.0 (SPSS, Chicago, IL, USA) and R version 3.3.0 for Windows (https://www.R-project.org), and the packages 'forest', 'survival', 'Hmisc', 'ROCR' and 'mice'.

\section{Results}

Baseline characteristics From the source population $(n=4164), 496(11.9 \%)$ reported a diagnosis of diabetes mellitus at inclusion; of whom the vast majority $(92.5 \%)$ had type 2 diabetes. In total, 1107 (26.6\%) had single measurements of plasma glucose and/or $\mathrm{HbA}_{1 \mathrm{c}}$ suggestive of undiagnosed diabetes. Both plasma and urine KTR levels were elevated among participants with self-reported diabetes at study 
Table 1 Baseline characteristics of the prospective study population $(n=2519)$ according to incident type 2 diabetes

\begin{tabular}{|c|c|c|c|c|c|}
\hline \multirow[t]{2}{*}{ Variable } & \multirow[t]{2}{*}{ Valid $n$} & \multirow{2}{*}{$\begin{array}{l}\text { Total population } \\
n=2519\end{array}$} & \multicolumn{2}{|c|}{ Type 2 diabetes during follow-up } & \multirow[t]{2}{*}{$p$} \\
\hline & & & $\begin{array}{l}\text { No } \\
n=2346\end{array}$ & $\begin{array}{l}\text { Yes } \\
n=173\end{array}$ & \\
\hline Age (years) & 2519 & $61.3(10.4)$ & $61.4(10.4)$ & $62.3(10.3)$ & 0.74 \\
\hline Sex (men) & 2519 & $1841(73.1 \%)$ & $1714(73.1 \%)$ & $127(73.4 \%)$ & 0.92 \\
\hline Fasting & 642 & $642(25.5 \%)$ & $605(25.8 \%)$ & $37(21.4 \%)$ & 0.30 \\
\hline BMI $\left(\mathrm{kg} / \mathrm{m}^{2}\right)$ & 2519 & $25.9(3.71)$ & $25.7(3.56)$ & $28.1(3.69)$ & $<0.001$ \\
\hline Current smoking & 2519 & $809(32.1 \%)$ & $755(32.2 \%)$ & $54(31.2 \%)$ & 0.79 \\
\hline Hypertension & 2519 & $1114(44.2 \%)$ & $1003(42.8 \%)$ & $111(64.2 \%)$ & $<0.001$ \\
\hline Systolic BP (mmHg) & 2491 & $139(125-152)$ & $139(125-152)$ & $140(126-154)$ & 0.27 \\
\hline Diastolic BP (mmHg) & 2490 & $80(75-88)$ & $80(75-88)$ & $83(76-90)$ & 0.01 \\
\hline Significant $C A D^{\mathrm{a}}$ & 2519 & $1888(75.0 \%)$ & $1744(74.3 \%)$ & $144(83.2 \%)$ & 0.01 \\
\hline \multicolumn{6}{|l|}{ Renal function and inflammation } \\
\hline Serum creatinine $(\mu \mathrm{mol} / \mathrm{l})$ & 2516 & 89 (81-98) & $89(81-98)$ & $91(83-100)$ & 0.26 \\
\hline eGFR $\left(\mathrm{ml} \mathrm{min} \min ^{-1} 1.73 \mathrm{~m}^{-2}\right)$ & 2516 & $91(79-99)$ & $91(79-99)$ & $91(77-99)$ & 0.71 \\
\hline Serum CRP $(\mathrm{nmol} / \mathrm{l})$ & 2519 & $16.1(7.90-32.4)$ & $15.8(7.81-31.9)$ & $21.1(10.3-37.0)$ & 0.02 \\
\hline Plasma neopterin (nmol/1) & 2504 & $8.09(6.65-10.1)$ & $8.09(6.65-10.1)$ & $8.11(6.50-10.0)$ & 0.81 \\
\hline Plasma kynurenine (nmol/1) & 2516 & $1.67(1.38-1.98)$ & $1.66(1.38-1.97)$ & $1.75(1.51-2.11)$ & 0.001 \\
\hline Plasma tryptophan $(\mu \mathrm{mol} / \mathrm{l})$ & 2516 & $70.3(61.4-79.5)$ & $70.1(61.3-79.2)$ & $72.5(62.2-82.6)$ & 0.01 \\
\hline Plasma KTR (nmol/ $/ \mu \mathrm{mol})$ & 2516 & $23.6(19.7-28.5)$ & $23.5(19.7-28.5)$ & $23.7(20.2-28.8)$ & 0.18 \\
\hline \multicolumn{6}{|l|}{ Serum lipids } \\
\hline ApoA-1 (g/l) & 2519 & $1.31(1.14-1.48)$ & $1.31(1.14-1.49)$ & $1.24(1.11-1.44)$ & 0.01 \\
\hline $\operatorname{ApoB}(g / l)$ & 2519 & $0.87(0.73-1.05)$ & $0.87(0.73-1.04)$ & $0.91(0.76-1.08)$ & 0.07 \\
\hline Triacylglycerol (mmol/l) & 2516 & $1.44(1.06-2.03)$ & $1.41(1.04-2.00)$ & $1.70(1.25-2.56)$ & $<0.001$ \\
\hline \multicolumn{6}{|l|}{ Glucose homeostasis } \\
\hline Plasma glucose $(\mathrm{mmol} / \mathrm{l})$ & 2518 & $5.4(5.0-6.1)$ & $5.4(5.0-6.0)$ & $6.3(5.7-7.8)$ & $<0.001$ \\
\hline $\mathrm{HbA}_{1 \mathrm{c}}(\%)$ & 2519 & $5.6(5.0-6.0)$ & $5.6(5.0-6.0)$ & $5.7(5.1-6.1)$ & 0.16 \\
\hline $\mathrm{HbA}_{1 \mathrm{c}}(\mathrm{mmol} / \mathrm{mol})$ & 2519 & $38(31-42)$ & $38(31-42)$ & $39(32-43)$ & - \\
\hline Serum insulin $(\mathrm{pmol} / \mathrm{l})$ & 607 & $21.8(19.7-55.0)$ & $19.7(19.7-55.0)$ & $39.4(19.7-110)$ & 0.002 \\
\hline Serum C-peptide (nmol/l) & 607 & $0.71(0.53-0.98)$ & $0.71(0.51-0.96)$ & $0.91(0.67-1.17)$ & 0.001 \\
\hline \multicolumn{6}{|l|}{ HOMA2 C-peptide } \\
\hline Beta cell activity & 607 & $113(93-138)$ & $112(92-138)$ & $121(95-149)$ & 0.57 \\
\hline Insulin resistance & 607 & $1.6(1.2-2.2)$ & $1.6(1.1-2.2)$ & $2.0(1.5-2.7)$ & $<0.001$ \\
\hline \multicolumn{6}{|l|}{ Urine biomarkers } \\
\hline Creatinine $(\mathrm{mmol} / \mathrm{l})$ & 2263 & $11.7(7.8-16.6)$ & $11.8(7.8-16.6)$ & $11.3(8.3-16.4)$ & 0.77 \\
\hline Albumin:creatinine (mg/mmol) & 2111 & $0.51(0.37-0.82)$ & $0.50(0.37-0.81)$ & $0.60(0.42-0.99)$ & 0.02 \\
\hline Kynurenine:creatinine $(\mathrm{nmol} / \mathrm{mmol})$ & 2263 & $182(118-277)$ & $179(117-275)$ & $221(151-322)$ & 0.001 \\
\hline Tryptophan:creatinine $(\mu \mathrm{mol} / \mathrm{mmol})$ & 2263 & $4.86(3.68-6.47)$ & $4.82(3.68-6.46)$ & $5.32(3.70-6.75)$ & 0.43 \\
\hline FE of kynurenine ${ }^{b}$ & 2260 & $8.25(5.42-12.1)$ & $8.15(5.36-12.1)$ & $9.52(6.29-13.0)$ & 0.03 \\
\hline FE of tryptophan ${ }^{\mathrm{b}}$ & 2260 & $5.27(3.81-7.08)$ & $5.27(3.81-7.07)$ & $5.28(3.83-7.26)$ & 0.80 \\
\hline Urine KTR (nmol/ $\mu \mathrm{mol})$ & 2263 & $36.3(27.7-49.4)$ & $36.2(27.5-48.9)$ & $39.8(31.3-61.1)$ & $<0.001$ \\
\hline \multicolumn{6}{|l|}{ Medications } \\
\hline Aspirin & 2519 & $2084(82.7 \%)$ & $1937(82.6 \%)$ & $147(85.0 \%)$ & 0.42 \\
\hline Statins & 2519 & $2020(80.2 \%)$ & $1877(80.0 \%)$ & $143(82.7 \%)$ & 0.40 \\
\hline$\beta$-blockers & 2519 & $1830(72.6 \%)$ & $1696(72.3 \%)$ & $134(77.5 \%)$ & 0.14 \\
\hline Loop diuretics & 2519 & $231(9.2 \%)$ & $205(8.7 \%)$ & $26(15.0 \%)$ & 0.006 \\
\hline Thiazides & 2519 & $159(6.3 \%)$ & $141(6.0 \%)$ & $18(10.4 \%)$ & 0.02 \\
\hline ACE inhibitors and/or ARB & 2519 & $723(28.7 \%)$ & $643(27.4 \%)$ & $80(46.2 \%)$ & $<0.001$ \\
\hline
\end{tabular}

Data are presented as $n(\%)$, mean (SD) or median (IQR)

${ }^{\text {a }}$ At least one stenosis with $\geq 50 \%$ luminal narrowing in a main coronary artery or its major side branches identified by coronary angiography

${ }^{\mathrm{b}} \mathrm{FE}=\left([\text { kynurenine }]_{\text {urine }} \times[\text { creatinine }]_{\text {plasma }}\right) /\left([\text { kynurenine }]_{\text {plasma }} \times[\text { creatinine }]_{\text {urine }}\right)$

$\mathrm{ARB}$, angiotensin II receptor blockers

enrolment. Other characteristics according to diabetes mellitus status at baseline are given in ESM Table 1.

Of the 2519 participants included in the prospective analyses, 1841 (73.1\%) were men and 1888 (75.0\%) had significant $\mathrm{CAD}$ at coronary angiography. The mean (SD) age was $61.3(10.4)$ years and mean BMI was 25.9 (3.71) $\mathrm{kg} / \mathrm{m}^{2}$. A total of $642(25.5 \%)$ participants reported to be fasting at the time of sampling, with no differences in plasma or urine KTR levels according to fasting status (data not shown). 
Compared with those without a diagnosis of type 2 diabetes during follow-up, participants who developed type 2 diabetes had higher BMI, and higher prevalences of hypertension and significant CAD at baseline. They were also more frequently receiving thiazides and loop diuretics. Median serum levels of CRP, triacylglycerol, plasma glucose and HOMA-IR were higher among those who developed type 2 diabetes, whereas ApoA-1 levels were lower (Table 1). Although numerically slightly higher among those with incident type 2 diabetes, neither the difference in baseline $\mathrm{HbA}_{1 \mathrm{c}}$ levels nor the difference in plasma KTR or neopterin levels were statistically significant. In contrast, those who subsequently developed type 2 diabetes had substantially higher levels of urine KTR $(p<0.001)$. They also had higher urine kynurenine:creatinine ratios and $\mathrm{FE}$ of kynurenine; however, the tryptophan:creatinine ratios and FE of tryptophan were similar between the groups (Table 1).

Covariates associated with plasma and urine KTR In bivariate analyses, KTR in plasma and urine were strongly related $(\beta=0.45, p<0.001)$. Multivariate adjusted covariates of plasma and urine KTR are reported in Table 2. Both biomarkers were strongly positively associated with the IFN- $\gamma$ marker neopterin ( $\beta=0.46$ and $\beta=0.28$, respectively; both $p<0.001)$. Further, positive relationships were seen with age and CRP levels, whereas negative associations were found for
eGFR and ApoA-1. BMI and statin use were weakly positively associated with plasma KTR, but showed no significant relation to urine KTR. As shown in Table 2, sex, presence of $\mathrm{CAD}$, and the usage of loop diuretics, thiazides, ACE inhibitors, angiotensin II receptor blockers or $\beta$-blockers were not associated with KTR levels in urine or plasma. Furthermore, systolic or diastolic BP were not identified as independent covariates (data not shown). The total explained variances $\left(R^{2}\right)$ of all variables in Table 2 were $48 \%$ and $25 \%$ for plasma and urine KTR, respectively.

To evaluate the impact of insulin resistance on circulating and urinary levels of KTR, the linear regression analyses were repeated for a subset of participants for whom HOMA calculations were available ( $n=607$; data not shown). In a multivariate model including the same covariates as in Table 2, HOMA-IR was only weakly associated with plasma KTR ( $\beta=0.09, p=0.04)$ and showed no significant relationship to urine $\operatorname{KTR}(\beta=0.05, p=0.29)$.

KTR and risk of type 2 diabetes During a median (IQR) of $7.6(6.6-8.7)$ years, a total of $173(6.9 \%)$ new diagnoses of type 2 diabetes were identified. There was no significant association between plasma KTR and risk of type 2 diabetes (Fig. 1a). However, urine KTR was a strong predictor of type 2 diabetes (Fig. 1b). Comparing Q4 with Q1 of urine KTR, the

Table 2 Covariates associated with plasma $(n=2516)$ and urine $(n=2263)$ KTR in multivariate linear regression analyses

\begin{tabular}{|c|c|c|c|c|c|}
\hline \multirow[t]{2}{*}{ Covariate } & \multirow[t]{2}{*}{ SD } & \multicolumn{2}{|c|}{ Plasma $\left(\log _{\mathrm{e}}\right) \mathrm{KTR}^{\mathrm{a}}(\mathrm{nmol} / \mu \mathrm{mol})$} & \multicolumn{2}{|c|}{ Urine $\left(\log _{\mathrm{e}}\right) \mathrm{KTR}^{\mathrm{b}}(\mathrm{nmol} / \mu \mathrm{mol})$} \\
\hline & & $\beta^{\mathrm{c}}(95 \% \mathrm{CI})$ & $p$ & $\beta^{\mathrm{c}}(95 \% \mathrm{CI})$ & $p$ \\
\hline Age (years) & 10.4 & $0.12(0.08,0.16)$ & $<0.001$ & $0.15(0.11,0.20)$ & $<0.001$ \\
\hline Sex (men) & - & $-0.012(-0.046,0.022)$ & 0.50 & $0.024(-0.017,0.066)$ & 0.25 \\
\hline BMI $\left(\mathrm{kg} / \mathrm{m}^{2}\right)$ & 3.71 & $0.066(0.033,0.099)$ & $<0.001$ & $0.023(-0.017,0.063)$ & 0.26 \\
\hline eGFR (ml min $\left.\min ^{-1} 1.73 \mathrm{~m}^{-2}\right)$ & 0.24 & $-0.22(-0.26,-0.17)$ & $<0.001$ & $-0.125(-0.178,-0.072)$ & $<0.001$ \\
\hline $\mathrm{CAD}^{\mathrm{d}}$ (dichotomous) & - & $0.015(-0.022,0.052)$ & 0.43 & $0.021(-0.024,0.066)$ & 0.36 \\
\hline Urine albumin:creatinine ratio $(\mathrm{mg} / \mathrm{mmol})$ & 0.88 & $-0.009(-0.042,0.024)$ & 0.59 & $0.043(0.003,0.083)$ & 0.03 \\
\hline $\mathrm{HbA}_{1 \mathrm{c}}(\%)$ & 0.15 & $0.009(-0.022,0.041)$ & 0.56 & $0.006(-0.032,0.044)$ & 0.77 \\
\hline Triacylglycerol (mmol/l) & 0.50 & $-0.001(-0.034,0.032)$ & 0.95 & $-0.067(-0.107,-0.027)$ & 0.001 \\
\hline ApoA-1 (g/l) & 0.20 & $-0.094(-0.128,-0.059)$ & $<0.001$ & $-0.061(-0.103,-0.019)$ & 0.004 \\
\hline CRP (nmol/l) & 1.08 & $0.039(0.006,0.072)$ & 0.02 & $0.092(0.052,0.132)$ & $<0.001$ \\
\hline Neopterin (nmol/l) & 0.37 & $0.46(0.42,0.50)$ & $<0.001$ & $0.28(0.23,0.33)$ & $<0.001$ \\
\hline Use of statins & - & $0.047(0.013,0.082)$ & 0.007 & $0.013(-0.029,0.055)$ & 0.54 \\
\hline Use of loop diuretics & - & $0.011(-0.022,0.044)$ & 0.52 & $-0.028(-0.068,0.012)$ & 0.18 \\
\hline Use of thiazides & - & $-0.016(-0.049,0.018)$ & 0.36 & $-0.006(-0.046,0.033)$ & 0.75 \\
\hline Use of ACE inhibitors and/or ARB & - & $0.019(-0.016,0.053)$ & 0.28 & $0.0001(-0.041,0.041)$ & 0.99 \\
\hline Use of $\beta$-blocker & - & $-0.015(-0.048,0.019)$ & 0.38 & $-0.032(-0.073,0.008)$ & 0.12 \\
\hline
\end{tabular}

${ }^{\text {a }}$ Multiple $R^{2}: 48 \%$

${ }^{\mathrm{b}}$ Multiple $R^{2}: 25 \%$

${ }^{\mathrm{c}}$ Standardised $\beta$ coefficients

${ }^{d}$ At least one stenosis with $\geq 50 \%$ luminal narrowing in a main coronary artery or its major side branches identified by coronary angiography $\mathrm{ARB}$, angiotensin II receptor blockers 


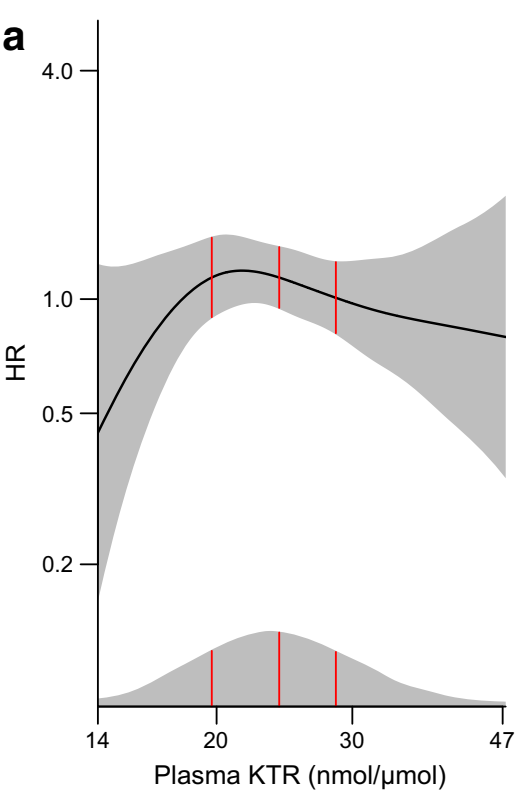

Fig. 1 Cox regression with penalised smoothing splines showing the associations of (a) plasma KTR and (b) urine KTR to incident type 2 diabetes $(n=2263)$. The solid lines denote HRs and the grey areas, 95\% CIs on the ( $\log _{\mathrm{e}}$-transformed) $y$-axes. Density plots show the distributions of ( $\log _{e}$ transformed) plasma and urine KTR concentrations on the $x$-axes. The vertical red lines mark the 25th, 50th and 75th percentiles. Both

HRs (95\% CIs) were $2.59(1.56,4.30)$ and $2.35(1.39,3.96)$ in the age- and sex-adjusted and multivariate models, respectively (Table 3). Repeating the Cox analyses using multiple multivariate imputation provided nearly identical results (ESM Table 2). In the subset for whom calculations of HOMA indices were available $(n=607)$, urine KTR predicted new onset type 2 diabetes even after adjustment for each of the

Table 3 HRs (95\% CIs) for incident type 2 diabetes mellitus by KTR in plasma $^{\mathrm{a}}(n=2516)$ and urine ${ }^{\mathrm{b}}(n=2263)$

\begin{tabular}{|c|c|c|c|c|}
\hline & \multicolumn{2}{|l|}{ Per SD increase } & \multicolumn{2}{|l|}{ Q4 vs Q1 } \\
\hline & $\mathrm{HR}(95 \% \mathrm{CI})$ & $p$ & $\mathrm{HR}(95 \% \mathrm{CI})$ & $p$ \\
\hline \multicolumn{5}{|c|}{ Plasma $\left(\log _{\mathrm{e}}\right) \mathrm{KTR}$} \\
\hline Model $1^{\mathrm{c}}$ & $1.14(0.97,1.33)$ & 0.11 & $1.27(0.80,2.03)$ & 0.31 \\
\hline Model $2^{\mathrm{d}}$ & $0.99(0.78,1.22)$ & 0.91 & $0.97(0.54,1.73)$ & 0.92 \\
\hline \multicolumn{5}{|c|}{ Urine $\left(\log _{e}\right)$ KTR } \\
\hline Model $1^{\mathrm{c}}$ & $1.39(1.19,1.62)$ & $<0.001$ & $2.59(1.56,4.30)$ & $<0.001$ \\
\hline Model $2^{\mathrm{d}}$ & $1.38(1.16,1.64)$ & $<0.001$ & $2.35(1.39,3.96)$ & 0.001 \\
\hline
\end{tabular}

${ }^{\mathrm{a}} \mathrm{SD}: 0.31 \mathrm{nmol} / \mu \mathrm{mol}$

${ }^{\mathrm{b}} \mathrm{SD}: 0.47 \mathrm{nmol} / \mu \mathrm{mol}$

${ }^{\mathrm{c}}$ Adjusted for age and sex

${ }^{\mathrm{d}}$ Adjusted for age, sex, BMI, eGFR, CRP, $\mathrm{HbA}_{1 \mathrm{c}}$, serum triacylglycerol, ApoA-1, urine albumin:creatinine ratio, and the use of loop diuretics, ACE inhibitors or angiotensin II receptor blockers, statins and $\beta$-blockers

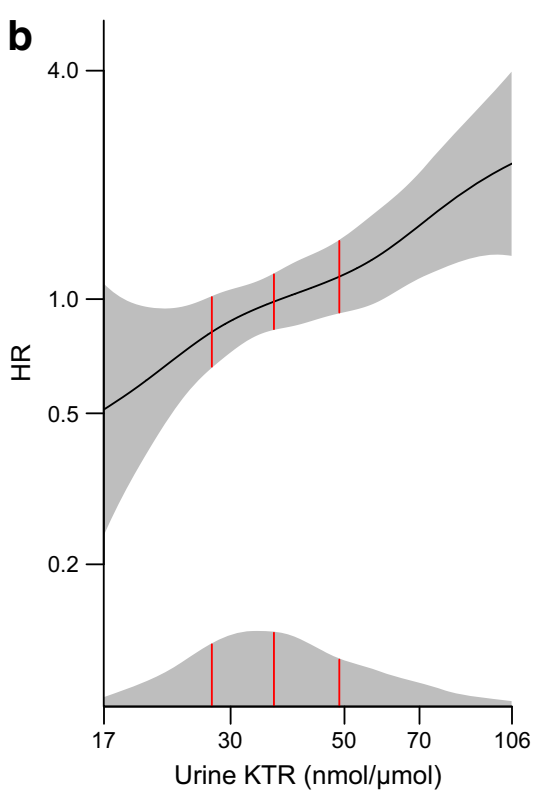

models include adjustments for age, sex, BMI, eGFR, CRP, $\mathrm{HbA}_{1 \mathrm{c}}$, serum triacylglycerol, ApoA-1, urine albumin:creatinine ratio, and use of loop diuretics, ACE inhibitors or angiotensin II receptor blockers, statins and $\beta$-blockers. Ranges from the 2.5 th to 97.5 th percentiles of exposure variables are included

following: HOMA-B, HOMA-IR, insulin and C-peptide (ESM Table 3).

In the total population, we also observed significant associations between the urine kynurenine:creatinine ratio as well as the FE of kynurenine with risk of incident type 2 diabetes (ESM Table 4). In contrast, no such relationships were seen for tryptophan:creatinine ratio or the FE of tryptophan (ESM Table 5).

Calculated by AIC, the addition of urine KTR to the multivariate model significantly improved goodness of fit. Moreover, urine KTR provided an NRI of 0.21 (0.04, 0.38; $p=0.02)$, as well as a significant increase in the $\mathrm{C}$ statistic $(p=0.04)($ ESM Table 6$)$.

Subgroup analyses The multivariate association of urine KTR to new onset type 2 diabetes was further evaluated in strata of traditional diabetes risk indicators and other potential effect modifiers. Interestingly, urine KTR was a stronger predictor in subgroups having BMI and serum triacylglycerol levels below the median (Fig. 2); however, the interaction terms were not statistically significant when adjusted for multiple comparisons ( $p_{\text {int }}=0.09$ and $p_{\text {int }}=0.10$ for BMI and serum triacylglycerol, respectively). Of note, urine KTR predicted incident type 2 diabetes both among those with and without angiographically verified $\mathrm{CAD}$ at baseline, with no significant effect modification according to CAD status (Fig. 2). 


$\begin{array}{llr} & & n \\ \text { Age (years) } & \geq 62.0 & 1078 \\ & <62.0 & 1027 \\ \text { Sex } & \text { Women } & 558 \\ & \text { Men } & 1547 \\ \text { BMI }\left(\mathrm{kg} / \mathrm{m}^{2}\right) & & \\ & \geq 26.0 & 1055 \\ \text { CAD } & <26.0 & 1050 \\ & \text { Yes } & 1606 \\ \text { eGFR }\left(\mathrm{ml} \text { min }^{-1} 1.73 \mathrm{~m}^{-2}\right) & \geq 91 & 499 \\ & <91 & 1068 \\ \text { CRP }(\mathrm{nmol} / \mathrm{l}) & & 1037 \\ & \geq 13.7 & 1044 \\ \text { HbA }{ }_{1 \mathrm{c}}(\mathrm{mmol} / \mathrm{mol},[\%]) & <13.7 & 1061 \\ & \geq 38,[5.6 \%] & 1033 \\ \text { Triacylglycerol }(\mathrm{mmol} / \mathrm{l}) & <38,[5.6 \%] & 1072 \\ & \geq 1.44 & 1045 \\ \text { ApoA-1 (g/l) } & <1.44 & 1060 \\ & \geq 1.31 & 1064 \\ \text { U-alb:creat }(\mathrm{mg} / \mathrm{mmol}) & <1.31 & 1041 \\ & \geq 0.50 & 1071 \\ & <0.50 & 1034 \\ & & \end{array}$

Fig. 2 Multivariate adjusted HRs of urine KTR for incident type 2 diabetes in subgroups. Dichotomous subgroups were generated for sex and significant $\mathrm{CAD}$ at coronary angiography (at least one stenosis with $\geq 50 \%$ luminal narrowing in a main coronary artery or its major side branches, identified by coronary angiography), or according to the median values of continuous variables. HRs are represented by squares and are

\section{Discussion}

Principal findings In a large cohort of individuals with CAD, we observed that KTR in urine, but not in plasma, was a strong predictor of incident type 2 diabetes during 7 years of follow-up. The risk relationship was similar after extensive adjustment for potential confounders. Moreover, urine KTR significantly improved model discrimination and risk classification, both features being prerequisites for the potential clinical usefulness of a biomarker. Notably, urine KTR was an even stronger predictor among participants at presumably lower risk of type 2 diabetes evaluated by BMI and serum triacylglycerol levels.

Strengths and limitations Major strengths of our study include the large sample size, prospective design with long follow-up time, and detailed descriptions of clinical and biochemical baseline characteristics. The information on incident type 2 diabetes was collected from national health registries to which reporting is mandatory for all drug prescriptions and hospital admissions in Norway. There may have been some under-reporting of new onset type 2 diabetes if individuals did not receive pharmacological intervention or were not admitted $p$ for interaction

0.92

0.91

0.009

0.43

0.83

0.26

0.36

0.02

0.12

0.79

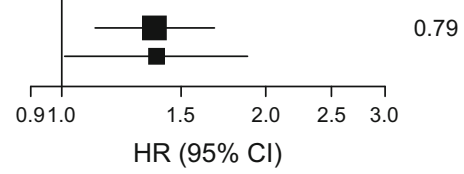

reported per SD increment of $\log _{e}$ transformed urine KTR levels. The horizontal lines indicate $95 \%$ CIs. HRs are adjusted for age, sex, BMI, eGFR, CRP, $\mathrm{HbA}_{1 \mathrm{c}}$, serum triacylglycerol, ApoA-1, urine albumin: creatinine (U-alb:creat) ratio, and use of loop diuretics, ACE inhibitors or angiotensin II receptor blockers, statins and $\beta$-blockers

to hospital during follow-up. Further, our study design did not permit the identification of individuals who actually had incident autoimmune diabetes. However, prior findings in northern European populations suggest that only $4 \%$ of individuals with newly diagnosed phenotypic type 2 diabetes may be GAD antibody positive [28]. Therefore, any misclassification is unlikely to have affected our results.

Unlike most other spot urine biomarkers, KTR does not require adjustment for urine creatinine to account for dilution. This limits the possibility of confounding, since the urinary creatinine excretion rate may be influenced by common comorbidities [29]. We have previously demonstrated good within-person reproducibility of urine KTR [19], which justifies single measurement of biomarker status [30]. Previous studies have suggested that urine tryptophan and kynurenine levels were stable in storage at room temperature for at least $48 \mathrm{~h}$, representing a practical advantage in a clinical setting [31].

The study participants were all referred to hospital for elective coronary angiography. The majority had CAD, which is frequently associated with insulin resistance [32]. Notably, however, urine KTR was a strong predictor even in the subgroup without angiographic evidence of CAD. Our findings 
nonetheless need to be confirmed in large population-based surveys. Unfortunately, a replication cohort for plasma and urine KTR could not be provided for the present study. Future work should also evaluate urinary levels of downstream metabolites of the kynurenine pathway. As in any observational study the risk of residual confounding cannot be excluded. Further, our work does not allow firm conclusions about pathophysiological mechanisms.

Tryptophan metabolism, type 2 diabetes and related morbidities High levels of plasma KTR have been associated with adverse cardiovascular prognoses [33], with consistent findings in individuals with CAD [33] and in elderly people [34]. Moreover, we previously identified urine KTR as a particularly strong predictor of coronary events and death [19]. In contrast, epidemiological data on the kynurenine pathway in relation to type 2 diabetes have been sparse. Obesity has been associated with elevated plasma KTR levels, which were not normalised even after profound weight loss [6]. A crosssectional study showed that, compared with healthy individuals, plasma KTR was increased in individuals with type 2 diabetes, and levels were even higher in those with diabetic nephropathy [35]. Moreover, urinary excretions of kynurenine [36], and its downstream metabolite xanthurenic acid, were elevated among individuals with type 2 diabetes [14]. A recent smaller case-control study reported an association of plasma tryptophan levels to new onset type 2 diabetes [37]. To the best of our knowledge, however, the relationships of plasma and urine KTR levels with incident type 2 diabetes have not previously been evaluated in a large-scale prospective setting.

Possible mechanisms More than 95\% of tryptophan metabolism occurs through the kynurenine pathway where the first and rate-limiting step is catalysed either by the hepatic enzyme tryptophan 2,3-dioxygenase (TDO) or the ubiquitous IDO1. While TDO is constitutively active, IDO1 is induced by IFN- $\gamma$ or other inflammatory cytokines. TDO is suppressed in parallel with IDO1 induction. Hence, in conditions with increased IFN- $\gamma$ activity, tryptophan metabolism is shifted from the liver to extrahepatic tissues [3].

IDO1 and genes for several downstream enzymes of the kynurenine pathway were upregulated in adipose tissue of obese compared with lean women [11] and were expressed both in adipocytes and macrophages [11]. IDO1 may also be induced in pancreatic beta cells [38], as well as renal glomerular [10, 39] and tubular cells [40]. The role of tryptophan metabolism through the TDO enzyme in diabetes development is not established. TDO was not found to be increased in obese individuals [41]. In contrast, a rodent model demonstrated highly upregulated TDO expression in diet-induced obesity [42]. In our cohort, plasma and urine KTR were strongly related with plasma neopterin, indicating that IFN- $\gamma$ mediated IDO1 activation is a major determinant for circulating, as well as urinary, KTR levels. However, this assumption needs to be confirmed in a properly designed experiment.

In line with previous cross-sectional findings [11, 19], we observed higher KTR levels in plasma and urine among participants with suspected or established diabetes mellitus at baseline. Further, plasma and urine KTR showed similar relationships to phenotypes of the MetS. However, in prospective analyses there was no significant association between circulating KTR levels and long-term risk of type 2 diabetes, whereas urine KTR was a strong predictor. Notably, urine KTR remained strongly associated with type 2 diabetes risk even after extensive adjustment for potential confounders.

The positive association of urine KTR with incident type 2 diabetes seems primarily to reflect elevated urinary excretion of kynurenine. The FE of tryptophan showed no significant association to type 2 diabetes development. According to previous findings, the renal elimination of kynurenine depends strongly on circulating levels [43]. Kynurenine is freely filtered in the glomeruli and undergoes nearly $100 \%$ tubular reabsorption at very low plasma concentrations but is increasingly excreted at elevated plasma levels [43]. This may suggest that the clearance of kynurenine is tightly regulated in order to balance circulating concentrations. Hence, urine KTR may potentially represent a more sensitive indicator of systemic tryptophan degradation than the corresponding plasma biomarker, but it may also reflect renal catabolism of tryptophan. Interestingly, glomerular IDO1 expression was upregulated in a rodent model of type 2 diabetes [39].

Kynurenine is an endogenous ligand of the transcription factor aryl hydrocarbon receptor [13], which mediates proinflammatory and procoagulant effects [44]. IDO1 activation has cytotoxic effects on Th1 lymphocytes [8]. Further, a recent experimental model showed that acute exposure of pancreatic islets to kynurenine augmented glucose-stimulated insulin secretion [12]. Moreover, tryptophan depletion in the microenvironment has been shown to activate the general control nonderepressible-2 (GCN2) kinase [45], which protects human glomerular endothelial cells from excessive glucose influx [46].

The kidneys are centrally involved in the pathogenesis of endothelial dysfunction [47]. Impaired nitric oxide (NO) mediated arterial vasodilation is a hallmark of the MetS and type 2 diabetes [48] as well as of cardiovascular disease (CVD) [48]. Interestingly, kynurenine has been identified as a potent endothelium-derived vasodilator, acting independently of NO [10]. Hence, elevated renal kynurenine excretion may adversely affect renal and systemic vascular function. This may partly explain the strong prognostic information from urine KTR in relation to incident type 2 diabetes in the current study, as well as to CVD, as previously shown [19].

Clinical implications We revealed a strong and $\log _{\mathrm{e}}$-linear association of urine KTR with incident type 2 diabetes several 
years before the development of clinical disease. Prevalence data suggest that type 2 diabetes is undetected in at least $30 \%$ of cases [49]. Moreover, the development of micro- and macrovascular complications may precede the progression to overt type 2 diabetes [49]. However, several of the established risk factors for type 2 diabetes are only weakly associated with vascular events [50]. Our findings that urine KTR predicted both CVD prognosis [19] and type 2 diabetes, as well as the strong risk association among individuals not being identified by classical risk factors, encourage its further evaluation with regards to clinical application.

Conclusions In a large prospective cohort study of individuals with suspected or verified CAD, urine KTR was a strong predictor of incident type 2 diabetes. The roles of the tryptophan degradation pathway and renal kynurenine excretion in type 2 diabetes development should be further elucidated.

Acknowledgements We thank all the recruiting physicians and nurses, laboratory personnel and other coworkers at Haukeland University Hospital, Bergen, Norway; Stavanger University Hospital, Stavanger, Norway; and Bevital, Bergen, Norway. The authors are indebted to T. Dimoski at the Norwegian Knowledge Centre for the Health Services, Oslo, Norway, for his contribution by developing the software necessary for obtaining data from Norwegian hospitals, conducting the data collection and quality assurance of data in this project.

Data availability The datasets analysed during the current study are available from the corresponding author on reasonable request.

Funding The study was funded by the Norwegian Health Association and the Norwegian Extra Foundation for Health and Rehabilitation through EXTRA funds (grant number: 2014/FOM5642), the Norwegian Heart and Lung Patient Organisation, the Norwegian Ministry of Health and Care Services, the Western Norway Regional Health Authority, the Kristian Gerhard Jebsen Foundation, and the Department of Heart Disease, Haukeland University Hospital, Bergen, Norway. None of the study sponsors were involved in study design, data collection, analysis and interpretation of data, writing, or in the decision to submit the paper.

Duality of interest The authors declare that there is no duality of interest associated with this manuscript.

Contribution statement EWR and ERP analysed and interpreted data, and wrote the manuscript. ES, GFTS, MHEC, GST, GM and PRN made substantial contributions to analysis and interpretation of data. PMU and $\varnothing \mathrm{M}$ supervised the analyses of tryptophan and kynurenine and interpreted data. ERP, GFTS and OKN collected and processed clinical data. OKN and ERP conceived and designed the study. ERP and GST contributed to collecting and linking data registries for endpoint data. All authors edited/revised the manuscript. EWR is the guarantor of this work and, as such, had full access to all the data in the study and takes responsibility for the integrity of the data and the accuracy of the data analysis. All authors have approved the final version of the article.

Open Access This article is distributed under the terms of the Creative Commons Attribution 4.0 International License (http:// creativecommons.org/licenses/by/4.0/), which permits unrestricted use, distribution, and reproduction in any medium, provided you give appropriate credit to the original author(s) and the source, provide a link to the Creative Commons license, and indicate if changes were made.

\section{References}

1. Kahn SE, Cooper ME, Del Prato S (2014) Pathophysiology and treatment of type 2 diabetes: perspectives on the past, present, and future. Lancet 383:1068-1083

2. Donath MY, Shoelson SE (2011) Type 2 diabetes as an inflammatory disease. Nat Rev Immunol 11:98-107

3. Oxenkrug GF (2010) Metabolic syndrome, age-associated neuroendocrine disorders, and dysregulation of tryptophan-kynurenine metabolism. Ann N Y Acad Sci 1199:1-14

4. Schrocksnadel K, Wirleitner B, Winkler C, Fuchs D (2006) Monitoring tryptophan metabolism in chronic immune activation. Clin Chim Acta 364:82-90

5. Oxenkrug GF (2011) Interferon-gamma-inducible kynurenines/ pteridines inflammation cascade: implications for aging and aging-associated psychiatric and medical disorders. J Neural Transm 118:75-85

6. Oxenkrug G (2011) Interferon-gamma-inducible inflammation: contribution to aging and aging-associated psychiatric disorders. Aging Dis 2:474-486

7. Oxenkrug G (2013) Insulin resistance and dysregulation of tryptophan-kynurenine and kynurenine-nicotinamide adenine dinucleotide metabolic pathways. Mol Neurobiol 48:294-301

8. Mellor AL, Munn DH (2004) IDO expression by dendritic cells: tolerance and tryptophan catabolism. Nat Rev Immunol 4:762-774

9. Lapin IP (1976) Depressor effect of kynurenine and its metabolites in rats. Life Sci 19:1479-1483

10. Wang Y, Liu H, McKenzie G et al (2010) Kynurenine is an endothelium-derived relaxing factor produced during inflammation. Nat Med 16:279-285

11. Favennec M, Hennart B, Caiazzo R et al (2015) The kynurenine pathway is activated in human obesity and shifted toward kynurenine monooxygenase activation. Obesity 23:2066-2074

12. Liu JJ, Raynal S, Bailbe D et al (2015) Expression of the kynurenine pathway enzymes in the pancreatic islet cells. Activation by cytokines and glucolipotoxicity. Biochim Biophys Acta 1852:980-991

13. Oxenkrug G, Cornicelli J, van der Hart M, Roeser J, Summergrad P (2016) Kynurenic acid, an aryl hydrocarbon receptor ligand, is elevated in serum of Zucker fatty rats. Integr Mol Med 3:761-763

14. Oxenkrug GF (2015) Increased plasma levels of xanthurenic and kynurenic acids in type 2 diabetes. Mol Neurobiol 52:805-810

15. Oxenkrug G, Tucker KL, Requintina P, Summergrad P (2011) Neopterin, a marker of interferon-gamma-inducible inflammation, correlates with pyridoxal-5'-phosphate, waist circumference, HDLcholesterol, insulin resistance and mortality risk in adult Boston community dwellers of Puerto Rican origin. Am J Neuroprot Neuroregen 3:48-52

16. Oxenkrug GF, Turski WA, Zgrajka W, Weinstock JV, Summergrad P (2013) Tryptophan-kynurenine metabolism and insulin resistance in hepatitis C patients. Hepat Res Treat 2013:149247

17. Pedersen ER, Tuseth N, Eussen SJPM et al (2015) Associations of plasma kynurenines with risk of acute myocardial infarction in patients with stable angina pectoris. Arterioscler Thromb Vasc Biol $35: 455-462$

18. Cheng S, Rhee EP, Larson MG et al (2012) Metabolite profiling identifies pathways associated with metabolic risk in humans. Circulation 125:2222-2231 
19. Pedersen ER, Svingen GFT, Schartum-Hansen H et al (2013) Urinary excretion of kynurenine and tryptophan, cardiovascular events, and mortality after elective coronary angiography. Eur Heart J 34:2689-2696

20. Ebbing M, Bleie O, Ueland PM et al (2008) Mortality and cardiovascular events in patients treated with homocysteine-lowering B vitamins after coronary angiography: a randomized controlled trial. JAMA 300:795-804

21. Midttun O, Hustad S, Ueland PM (2009) Quantitative profiling of biomarkers related to B-vitamin status, tryptophan metabolism and inflammation in human plasma by liquid chromatography/tandem mass spectrometry. Rapid Commun Mass Spectrom 23:1371-1379

22. Rebnord EW, Pedersen ER, Strand E et al (2015) Glycated hemoglobin and long-term prognosis in patients with suspected stable angina pectoris without diabetes mellitus: a prospective cohort study. Atherosclerosis 240:115-120

23. Levy JC, Matthews DR, Hermans MP (1998) Correct homeostasis model assessment (HOMA) evaluation uses the computer program. Diabetes Care 21:2191-2192

24. Sulo G, Igland J, Vollset SE, Nygård O, Øyen N, Tell GS (2013) Cardiovascular disease and diabetes mellitus in Norway during 1994-2009 CVDNOR - a nationwide research project. Nor J Epidemiol 21:101-107

25. van Buuren S (2007) Multiple imputation of discrete and continuous data by fully conditional specification. Stat Methods Med Res $16: 219-242$

26. Hastie T, Tibshirani R (1986) Generalized additive models. Stat Sci 1:297-318

27. Pencina MJ, D'Agostino RB Sr, Steyerberg EW (2011) Extensions of net reclassification improvement calculations to measure usefulness of new biomarkers. Stat Med 30:11-21

28. Zinman B, Kahn SE, Haffner SM, O’Neill MC, Heise MA, Freed MI (2004) Phenotypic characteristics of GAD antibody-positive recently diagnosed patients with type 2 diabetes in North America and Europe. Diabetes 53:3193-3200

29. Ix JH, de Boer IH, Wassel CL, Criqui MH, Shlipak MG, Whooley MA (2010) Urinary creatinine excretion rate and mortality in persons with coronary artery disease: the Heart and Soul Study. Circulation 121:1295-1303

30. Clarke R, Shipley M, Lewington S et al (1999) Underestimation of risk associations due to regression dilution in long-term follow-up of prospective studies. Am J Epidemiol 150:341-353

31. Geeraerts F, Schimpfessel L, Crokaert R (1980) The stability of tryptophan metabolites prior to urine analysis. Clin Chim Acta 102:247-251

32. Haffner SM (1999) Epidemiology of insulin resistance and its relation to coronary artery disease. Am J Cardiol 84:11 J-14J

33. Pedersen ER, Midttun O, Ueland PM et al (2011) Systemic markers of interferon-gamma-mediated immune activation and long-term prognosis in patients with stable coronary artery disease. Arterioscler Thromb Vasc Biol 31:698-704

34. Sulo G, Vollset SE, Nygård $O$ et al (2013) Neopterin and kynurenine-tryptophan ratio as predictors of coronary events in older adults, the Hordaland Health Study. Int J Cardiol 168:14351440
35. Zhang Y, Ruan Y, Zhang P, Wang L (2016) Increased indoleamine 2,3-dioxygenase activity in type 2 diabetic nephropathy. J Diabetes Complicat 31:223-227

36. Fu H, Liu X, Yu W, Zhu L, Zheng D, Wang J (2013) Screening of urinary biomarkers in patients with type 2 diabetes mellitus. Wei Sheng Yan Jiu 42:907-914

37. Chen T, Zheng X, Ma X et al (2016) Tryptophan predicts the risk for future type 2 diabetes. PLoS One 11:e162192

38. Sarkar SA, Wong R, Hackl SI et al (2007) Induction of indoleamine 2,3-dioxygenase by interferon-gamma in human islets. Diabetes 56: $72-79$

39. Baban B, Liu JY, Mozaffari S (2013) Endoplasmic reticulum stress response and inflammatory cytokines in type 2 diabetic nephropathy: role of indoleamine 2,3-dioxygenase and programmed death-1. Exp Mol Pathol 94:343-351

40. Mohib K, Guan Q, Diao H, Du C, Jevnikar AM (2007) Proapoptotic activity of indoleamine 2,3-dioxygenase expressed in renal tubular epithelial cells. Am J Physiol Renal Physiol 293: F801-F812

41. Wolowczuk I, Hennart B, Leloire A et al (2012) Tryptophan metabolism activation by indoleamine 2,3-dioxygenase in adipose tissue of obese women: an attempt to maintain immune homeostasis and vascular tone. Am J Physiol Regul Integr Comp Physiol 303: R135-R143

42. Poulain-Godefroy O, Eury E, Leloire A et al (2013) Induction of $\mathrm{TDO} 2$ and IDO2 in liver by high-fat feeding in mice: discrepancies with human obesity. Int J Tryptophan Res 6:29-37

43. Moller SE (1981) Pharmacokinetics of tryptophan, renal handling of kynurenine and the effect of nicotinamide on its appearance in plasma and urine following L-tryptophan loading of healthy subjects. Eur J Clin Pharmacol 21:137-142

44. Sallee M, Dou L, Cerini C, Poitevin S, Brunet P, Burtey S (2014) The aryl hydrocarbon receptor-activating effect of uremic toxins from tryptophan metabolism: a new concept to understand cardiovascular complications of chronic kidney disease. Toxins 6:934 949

45. Eleftheriadis T, Pissas G, Antoniadi G, Liakopoulos V, Stefanidis I (2016) Indoleamine 2,3-dioxygenase depletes tryptophan, activates general control non-derepressible 2 kinase and down-regulates key enzymes involved in fatty acid synthesis in primary human CD4+ T cells. Immunology 146:292-300

46. Eleftheriadis T, Tsogka K, Pissas G, Antoniadi G, Liakopoulos V, Stefanidis I (2016) Activation of general control nonderepressible 2 kinase protects human glomerular endothelial cells from harmful high-glucose-induced molecular pathways. Int Urol Nephrol 48: 1731-1739

47. Endemann DH, Schiffrin EL (2004) Endothelial dysfunction. J Am Soc Nephrol 15:1983-1992

48. Huang PL (2009) eNOS, metabolic syndrome and cardiovascular disease. Trends Endocrinol Metab 20:295-302

49. Ryden L, Grant PJ, Anker SD et al (2013) ESC guidelines on diabetes, pre-diabetes, and cardiovascular diseases developed in collaboration with the EASD. Eur Heart J 34:3035-3087

50. Sattar N, Wannamethee SG, Forouhi NG (2008) Novel biochemical risk factors for type 2 diabetes: pathogenic insights or prediction possibilities? Diabetologia 51:926-940 